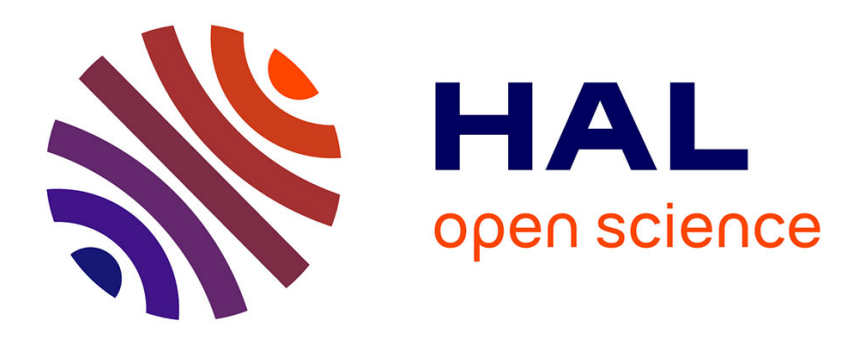

\title{
Differential method for freeform optics applied to two-mirror off-axis telescope design
}

\author{
Jean-Baptiste Volatier, Guillaume Druart
}

\section{To cite this version:}

Jean-Baptiste Volatier, Guillaume Druart. Differential method for freeform optics applied to twomirror off-axis telescope design. Optics Letters, 2019, 44 (5), pp.1174-1177. 10.1364/OL.44.001174 . hal-02317596

\section{HAL Id: hal-02317596 \\ https://hal.science/hal-02317596}

Submitted on 16 Oct 2019

HAL is a multi-disciplinary open access archive for the deposit and dissemination of scientific research documents, whether they are published or not. The documents may come from teaching and research institutions in France or abroad, or from public or private research centers.
L'archive ouverte pluridisciplinaire HAL, est destinée au dépôt et à la diffusion de documents scientifiques de niveau recherche, publiés ou non, émanant des établissements d'enseignement et de recherche français ou étrangers, des laboratoires publics ou privés. 


\title{
Differential method for freeform optics applied to two-mirror off-axis telescope design
}

\author{
Jean-Baptiste Volatier ${ }^{1, *}$ and Guillaume Druart ${ }^{1}$ \\ ${ }^{1}$ ONERA, Chemin de la Hunière, 91761 Palaiseau Cedex, France \\ ${ }^{*}$ Corresponding author: jean-baptiste.volatier@onera.fr
}

\begin{abstract}
In this letter we present a method to design the freeform surfaces of an off-axis unobscured two-mirror telescope by integration of a system of differential equations. The system is derived from the differentiation of the Fermat path's principle and is integrated as an ordinary differential equation problem. The method is used to design the freeform surfaces of a telescope whose performance is verified in off-the-shelf optical design software (Zemax).
\end{abstract}

Freeform optics are optical surfaces without axial symmetry which have been shown to improve optical systems performance. State-of-the-art freeform optical design methods rely on numerical optimization to find the optimum values of freeform description coefficients (Zernike, Forbes, RBF, splines), as such they suffer from the difficulties of inverse problem solving. For example, numerical optimization algorithms may converge to a local optimum of the performance merit function and the final result depends on the design chosen as a starting point. Global optimization methods are often not practical in freeform optical design because of the large solution space. Nonetheless, methods have been propose recently to mitigate these difficulties with strategies to find smart starting points [1] or analytic differentiation of optical design merit functions [2].

Other methods to design freform systems exist which take a direct approach by constructing the freeform surfaces from discrete rays. In illumination, if the light source can be considered punctual, each point on the optical surface can be uniquely associated with one ray, therefore it is possible to deduce for each point an optimum location and surface normal and to construct the freeform in that way [3]. Though in imaging (our problem of interest), the source cannot be considered punctual (extended object) so it is challenging to find the optimum location and surface normal because a dense set of rays will intersect the surface at the same point. Inconsistent results may occur, for example multiple surface normal orientations at the same point.

We broadly classify imaging construction methods by how they ensure the consistency of the freeform surfaces. First, simultaneous multiple surfaces (SMS) based methods $[4,5,6]$ use a small bundle of construction rays which are cleverly coupled to design consistent surfaces. Secondly, point-by-point methods $[7,8]$ use a dense batch of feature rays and fitting methods to recalculate iteratively surfaces normals. Third, methods have been developed $[9,10,11]$ which use differential information to extrapolate from a known ray to the optical system, they are however not freeform, since they only consider axial symmetric two-mirror systems and construct aspherical profiles (with axial symmetry).

In this letter we are particularly interested in the method described by Wassermann and Wolf [9]. A distinctive point is that it guarantees simultaneously axial stigmatism and exact satisfaction of the sine condition by designing two aspherical profiles, therefore subsequent numerical optimization of the system is not always necessary. Other distinctive characteristics are that both surfaces are simultaneously designed without iteration (contrary to point-by-point methods) and that rays are considered one-by-one (SMS considers small bundles of rays, while point-by-point use a large batch of feature rays).

This method deduces differential information by imposing that the optical system fulfills the sine condition, therefore even if all the constructed rays originate from the on-axis field point the degradation of 
performance in a field of view (FoV) close to the axis is controlled. From the on-axis ray a dense sampling of rays is constructed by numerical integration from the center of the pupil to its edge. The surfaces profiles are defined by the rays' intersection and are obtained as series of tabulated sag values. Another derivation of these equations, allowing analytical integration, was described later in literature [10, 11].

This method may not have seen widespread adoption by the optical design community due to two factors: the limitation to two optical surfaces and to axial symmetric systems. In this letter we will address the last point; future work will consider multiple surfaces. In our case, we control the performance in an extended field-of-view by imposing constraints derived from a differentiation of Fermat's path principle. These differential constraints are advantageous compared to the sine condition because they are suitable for the representation of a system without symmetry. The freeform surfaces obtained as tabulated values are then interpolated to model the surface in off-the-shelf optical design software as a mean of cross-validation by classical numerical ray tracing.

We first introduce a vector $\phi$ defined on $\mathbb{R}^{4}$ uniquely identifying rays. Since we consider a reflective infinite-to-finite conjugate system in this letter, we ignore the wavelength dependency and decompose $\boldsymbol{\phi}$ in four scalars $\left(k_{x}, k_{y}, p_{x}, p_{y}\right)$. The orientation of the ray is determined by the vector $\mathbf{k}=\left(k_{x}, k_{y}\right)$ and its intersection with the pupil of the system at a point identified by $\mathbf{p}=\left(p_{x}, p_{y}\right)$. We also define $\mathbf{P}_{\mathbf{i}}$ the intersection point $\left(X_{i}, Y_{i}, Z_{i}\right)$ of a ray with an optical surface $i$ in a global coordinate system. A ray is then represented by a sequence of segments between the points $\mathbf{P}_{\mathbf{0}}, \ldots, \mathbf{P}_{\mathbf{N}}$ depending on $\boldsymbol{\phi}$.

The goal of the following derivation is to compute the Taylor expansion coefficients of the functions characterizing the dependency of the rays as a function of $\boldsymbol{\phi}$. Doing so will allow us to extrapolate by numerical integration from the parabasal ray (defined for $\phi=(0,0,0,0)$ ) to the entire pupil and then to construct the freeform profiles. We set the propagation of the parabasal ray according to Figure 1 and consequently the origin of the pupil and field coordinates. These values are defined by the designer and the freeform profiles are derived from them, this is a limitation of the method described here: it is dependent on the parabasal ray configuration chosen by the designer. In our example we chose these values to compute a very compact telescope whose form factor would be appropriate for a micro-satellite. We choose $k_{x}=\tan \theta_{x}$ and $k_{y}=\tan \theta_{y}$ where $\theta_{x}$ and $\theta_{y}$ are the angles between the parabasal ray and the incoming ray projected respectively on the $x, z$ and $y, z$ planes.

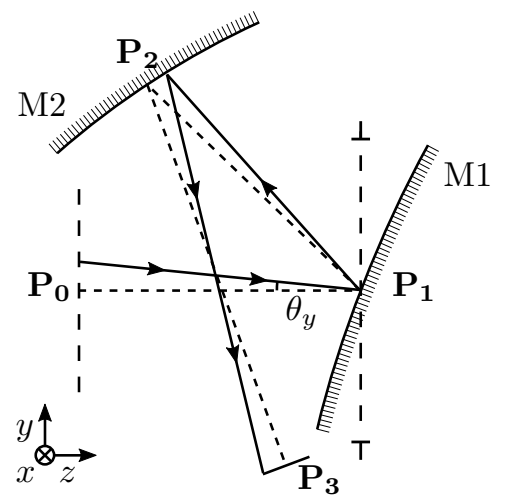

\begin{tabular}{lcc}
\hline$\#$ & $Y[\mathrm{~mm}]$ & $Z[\mathrm{~mm}]$ \\
\hline $\mathbf{P}_{\mathbf{0}}$ & 0 & 0 \\
$\mathbf{P}_{\mathbf{1}}$ & 0 & 80 \\
$\mathbf{P}_{\mathbf{2}}$ & 60 & 20 \\
$\mathbf{P}_{\mathbf{3}}$ & -50 & 60 \\
\hline
\end{tabular}

Figure 1: Parabasal ray $\boldsymbol{\phi}=(0,0,0,0)$ is drawn as a dashed line. A ray $\boldsymbol{\phi}=\left(0, \tan \theta_{y}, 0,0\right)$ is drawn as a solid line, showing that the system pupil is a plane intersecting M1 and parallel to the $x, y$ plane, this is a design choice suitable for this particular system. $\mathbf{P}_{\mathbf{0}}$ is on a dummy plane $Z_{0}=0$ and $\mathbf{P}_{\mathbf{3}}$ is on the image plane, $X$ values are all zero.

The optical system requirements are a focal length of $150 \mathrm{~mm}$ and an entrance pupil diameter of $70 \mathrm{~mm}$ with earth observation from micro satellites in the infrared $(8-12 \mu \mathrm{m})$ as intended application. Our requirement is a root-mean-square (RMS) geometrical spot-size better than $15 \mu \mathrm{m}$ over a field of $4 \times 0.4^{\circ}$.

Since $\mathbf{P}_{\mathbf{i}}$ is a point on a surface, there also exists a vector $\mathbf{w}_{\mathbf{i}}$ in $\mathbb{R}^{2}$ such that a function $\mathbf{w}_{\mathbf{i}} \mapsto \mathbf{P}_{\mathbf{i}}$ exist which in turn imposes restrictions on the partial derivatives of $\mathbf{P}_{\mathbf{i}}$ with respect to $\boldsymbol{\phi}$. Taking as an example 
the jacobian matrix, by application of the chain rule, the following decomposition holds:

$$
\frac{\partial \mathbf{P}_{\mathbf{i}}}{\partial \phi}=\frac{\partial \mathbf{P}_{\mathbf{i}}}{\partial \mathbf{w}_{\mathbf{i}}} \frac{\partial \mathbf{w}_{\mathbf{i}}}{\partial \phi}
$$

As a consequence of Eq. (1), since for two matrices $\mathbf{A}, \mathbf{B}$, the rank of the dot product is such that $\operatorname{rank}(\mathbf{A B}) \leq \min (\operatorname{rank}(\mathbf{A}), \operatorname{rank}(\mathbf{B}))$, the jacobian matrix $\partial \mathbf{P}_{\mathbf{i}} / \partial \boldsymbol{\phi}$ is degenerated and of rank 2.

If we do not fulfill the constraint $\operatorname{rank}\left(\partial \mathbf{P}_{\mathbf{i}} / \partial \boldsymbol{\phi}\right) \leq 2$ the optical surfaces properties will be inconsistent. Introducing the vector $\mathbf{w}_{\mathbf{i}}$ allows us to take into account correctly the jacobian rank constraint and other constraints applicable to the hessian tensor and higher order derivatives of $\mathbf{P}_{\mathbf{i}}$. The vector $\mathbf{w}_{\mathbf{i}}$ does not need to correspond to a particular vector in a physical plane as its sole function is to express the fact that there is a $\mathbb{R}^{2}$ space which acts as a dimensional bottleneck between $\mathbb{R}^{4}$ the space of definition of $\phi$ and $\mathbb{R}^{3}$ the space of definition of $\mathbf{P}_{\mathbf{i}}$. Nonetheless, in this letter we will choose $\mathbf{w}_{\mathbf{i}}$ to be expressed in the global coordinate plane $x, y$ to simplify the calculus.

We recall that the Fermat path principle states that rays travel along a stationary path throughout the system. Let $L$ be the optical path throughout an optical system and $n_{i, i+1}$ the refractive index between surface $i$ and $i+1$ and $\left\|\mathbf{P}_{\mathbf{i}} \mathbf{P}_{\mathbf{i}+\mathbf{1}}\right\|$ is the Euclidean distance between $\mathbf{P}_{\mathbf{i}}$ and $\mathbf{P}_{\mathbf{i}+\mathbf{1}}$.

$$
L=\sum_{i=0}^{N} n_{i, i+1}\left\|\mathbf{P}_{\mathbf{i}} \mathbf{P}_{\mathbf{i}+\mathbf{1}}\right\|
$$

As a consequence of Fermat's path principle we have:

$$
\frac{\partial L}{\partial \mathbf{w}_{\mathbf{i}}}=\mathbf{0} \quad \forall i \in[1, N-1]
$$
$\mathbf{F}=\mathbf{0}$.

We define in Eq. (4), the vector $\mathbf{F}$ composed of the numerators of the $\partial L / \partial \mathbf{w}_{\mathbf{i}}$ terms so given Eq. (3),

$$
\begin{aligned}
\mathbf{F}_{\mathbf{i}} & =\left(\left\|\mathbf{P}_{\mathbf{i}-\mathbf{1}} \mathbf{P}_{\mathbf{i}}\right\|\left(X_{i}-X_{i+1}\right)+\left\|\mathbf{P}_{\mathbf{i}} \mathbf{P}_{\mathbf{i}+\mathbf{1}}\right\|\left(X_{i}-X_{i-1}\right)\right) \frac{\partial X_{i}}{\partial \mathbf{w}_{\mathbf{i}}} \\
& +\left(\left\|\mathbf{P}_{\mathbf{i}-\mathbf{1}} \mathbf{P}_{\mathbf{i}}\right\|\left(Y_{i}-Y_{i+1}\right)+\left\|\mathbf{P}_{\mathbf{i}} \mathbf{P}_{\mathbf{i}+\mathbf{1}}\right\|\left(Y_{i}-Y_{i-1}\right)\right) \frac{\partial Y_{i}}{\partial \mathbf{w}_{\mathbf{i}}} \\
& +\left(\left\|\mathbf{P}_{\mathbf{i}-\mathbf{1}} \mathbf{P}_{\mathbf{i}}\right\|\left(Z_{i}-Z_{i+1}\right)+\left\|\mathbf{P}_{\mathbf{i}} \mathbf{P}_{\mathbf{i}+\mathbf{1}}\right\|\left(Z_{i}-Z_{i-1}\right)\right) \frac{\partial Z_{i}}{\partial \mathbf{w}_{\mathbf{i}}} \\
\mathbf{F} & =\left[\mathbf{F}_{\mathbf{1}}, \ldots, \mathbf{F}_{\mathbf{i}}, \ldots, \mathbf{F}_{\mathbf{N}-\mathbf{1}}\right]
\end{aligned}
$$

In the considered geometry, $N=3$ so $\mathbf{F}$ is of dimension 4 . The vectors and matrices introduced and their dependencies are represented in Figure 2.

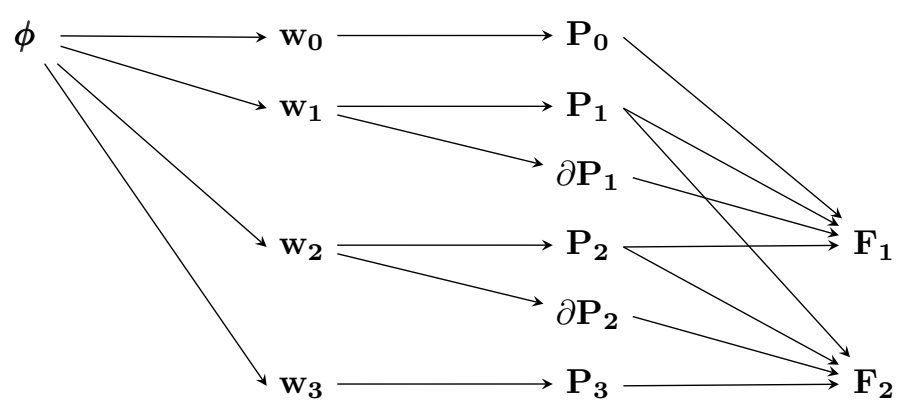

Figure 2: Vectors and matrices introduced and their dependencies. By $\partial \mathbf{P}_{\mathbf{i}}$ we imply the matrix $\partial \mathbf{P}_{\mathbf{i}} / \partial \mathbf{w}_{\mathbf{i}}$ 
Since $\mathbf{F}=\mathbf{0}$, the derivatives of $\mathbf{F}$ with respect to $\boldsymbol{\phi}$ are also zero. By application of the chain rule, we can set equations linking the derivatives of $\mathbf{F}$ and the derivatives of the functions representing the dependencies summarized in Figure 2. Solving these systems of equations allows us to compute the partial derivatives, some of the partial derivatives being known already.

The functions $\mathbf{w}_{\mathbf{i}} \mapsto \mathbf{P}_{\mathbf{i}}$ are known partially. We define $u_{i}, v_{i}$ subject to $\mathbf{w}_{\mathbf{i}}=\left(u_{i}, v_{i}\right)$ we deduce Eq. (5) from our earlier assumption that $\mathbf{w}_{\mathbf{0}}, \mathbf{w}_{\mathbf{1}}, \mathbf{w}_{\mathbf{2}}$ are expressed in the $x, y$ plane of Figure 1.

$$
\begin{aligned}
& \frac{\partial X_{i}}{\partial u_{i}}=\frac{\partial Y_{i}}{\partial v_{i}}=1 \\
& \frac{\partial X_{i}}{\partial v_{i}}=\frac{\partial Y_{i}}{\partial u_{i}}=0 \quad \forall i \in[0,1,2]
\end{aligned}
$$

The terms $\partial Z_{i} / \partial \mathbf{w}_{\mathbf{i}}$ are undetermined but can be derived according to Snell-Descartes law given that they are linked to the normal at the point of intersection since the matrix $\partial \mathbf{P}_{\mathbf{i}} / \partial \mathbf{w}_{\mathbf{i}}$ is a basis for the tangential plane at the same point.

The function $\phi \mapsto \mathbf{w}_{\mathbf{1}}$ is known since the surface 1 is the optical system pupil therefore $\mathbf{w}_{\mathbf{1}}=\mathbf{p}$.

The function $\phi \mapsto \mathbf{w}_{\mathbf{3}}$ is known at low order because we want to constraint paraxial properties and to correct for a set of aberrations. We set $f$ the focal length and we impose axial stigmatism and aplanetism to obtain Eq. (6).

$$
\begin{array}{ccc}
\frac{\partial u_{3}}{\partial k_{x}}=\frac{\partial v_{3}}{\partial k_{y}}=f & \text { and } & \frac{\partial u_{3}}{\partial k_{y}}=\frac{\partial v_{3}}{\partial k_{x}}=0 \\
\frac{\partial \mathbf{w}_{\mathbf{3}}}{\partial \mathbf{p}}=\mathbf{0} & \text { and } \quad & \frac{\partial^{2} \mathbf{w}_{\mathbf{3}}}{\partial \mathbf{k} \partial \mathbf{p}}=\mathbf{0}
\end{array}
$$

Higher order aberration terms like $\partial^{3} \mathbf{w}_{\mathbf{3}} / \partial \mathbf{k}^{2} \partial \mathbf{p}$ are not set to zero otherwise the system will be overconstrained. By allowing multiple surfaces and higher order differentiation, future work will allow to constrain these orders and to design anastigmats. There are similarities between our approach and the Taylor expansions used to derive aberration coefficients of freeform systems [12]. However we also establish relationships between aberrations and the derivatives of the freeform surfaces.

Finally, $\phi \mapsto \mathbf{w}_{\mathbf{0}}$ can be deduced from freespace propagation as a function of $Z_{1}$, surface 0 being a plane at $Z_{0}=0$.

The system of equations is set from $\partial \mathbf{F} / \partial \boldsymbol{\phi}=\mathbf{0}$ giving $4 \times 4$ equations and from $\partial^{2} \mathbf{F} / \partial \boldsymbol{\phi} \partial \mathbf{p}=\mathbf{0}$ giving $4 \times 4 \times 2$ equations. These two differentiations are necessary to take into account all the constraints set in Eq. (6). We thus obtain a system of 48 equations with 37 unknowns according to Table 1, column 1 . The derivatives $\partial Y_{3} / \partial v_{3}, \partial Z_{3} / \partial v_{3}$ are left undetermined by Eq. (5), so they are included in the 37 variables (line $\partial \mathbf{P}_{3}$ in Table 1). This way, we adjust the detector orientation while maintaining the vector $\partial \mathbf{P}_{\mathbf{3}} / \partial v_{3}$ normalized.

A software was developed leveraging the SymPy library [13], to perform the above differentiation automatically as required by the large operations count of the differentiated expressions (typically around 250 for one equation of $\partial \mathbf{F} / \partial \boldsymbol{\phi})$. Expressions of $\mathbf{F}$ are constructed from applications of UndefinedFunction SymPy objects. Differentiation of $\mathbf{F}$ produces new expressions containing Derivative objects. A merit function and its jacobian is then generated to evaluate the residuals of the system of equations and operates by substituting a vector of candidate values to the Derivative and UndefinedFunction applications objects, more details are given in Algorithm 1.

Once the optical surfaces are known at their intersection with the parabasal ray, we construct the full optical system by integration over the pupil. To maintain the consistency of the surfaces we use high order derivatives at rays already known to deduce lower order derivatives for new rays, see Eq. (7) for an example.

$$
\left.\left.\frac{\partial^{2} Z_{1}}{\partial \mathbf{w}_{\mathbf{1}}^{2}}\right|_{p_{x}=\epsilon} \approx \frac{\partial^{2} Z_{1}}{\partial \mathbf{w}_{\mathbf{1}}^{2}}\right|_{p_{x}=0}+\left.\epsilon\left(\frac{\partial^{3} Z_{1}}{\partial \mathbf{w}_{\mathbf{1}}^{3}} \frac{\partial \mathbf{w}_{\mathbf{1}}}{\partial p_{x}}\right)\right|_{p_{x}=0}
$$

Applying the same reasoning to the variables marked integrated in the Table 1, column 2, we create an ordinary differential equation integration problem. At each integration step the 48 equations are solved 


\begin{tabular}{lcc}
\hline variable & parabasal & off-axis \\
\hline $\mathbf{P}_{\mathbf{0}, \ldots, \mathbf{3}}$ & set & integrated \\
$\partial Z_{1,2}$ & derived & derived \\
$\partial \mathbf{P}_{\mathbf{3}}$ & set or solved & constant \\
$\partial^{2} Z_{1,2}$ & solved & integrated \\
$\partial^{3} Z_{1,2}$ & solved & solved \\
$\partial^{1,2} \mathbf{w}_{\mathbf{0}, \mathbf{1}}$ & derived & derived \\
$\partial \mathbf{w}_{\mathbf{1}}$ & solved & integrated \\
$\partial^{2} \mathbf{w}_{\mathbf{1}}$ & solved & solved \\
$\partial \mathbf{w}_{\mathbf{3}}$ & set & integrated \\
$\partial^{2} \mathbf{w}_{\mathbf{3}}$ & set & set or solved \\
\hline
\end{tabular}

set defined as part of the system definition

integrated calculated by the ordinary differential equation solver from the higher order derivatives at the previous point derived solved in closed form

solved determined by the system of equations and numerical root-finding

set or solved like solved but some terms of the vector are set, see text for details

constant solved once on-axis and kept constant afterwards.

Denominators are omitted, we imply that the derivative is with respect to the entire vector $\mathbf{w}_{\mathbf{i}}$ or $\boldsymbol{\phi}$.

Table 1: Variables and their determination

numerically for new values supplied by the ordinary differential equation solver for the variables marked integrated. The degrees of freedom are the higher order derivatives as explained in Table 1. Compared to the on-axis resolution we add supplementary degrees of freedom (DoF) given in Eq. (8) for the off-axis resolution to compensate for the fewer overall DoF available. These DoFs allow more optical performance degradation in the field along the $k_{y}$ direction. We select the $k_{y}$ direction to take advantage of the asymmetric field of view specification. Physically, the system will not be aplanetic along all axes in the $k_{y}$ field direction.

$$
\text { additional } \operatorname{DoF}=\left\{\frac{\partial^{2} u_{3}}{\partial k_{y} \partial p_{x}}, \frac{\partial^{2} u_{3}}{\partial k_{y} \partial p_{y}}, \frac{\partial^{2} v_{3}}{\partial k_{y} \partial p_{x}}\right\}
$$

We need an entrance pupil of $70 \mathrm{~mm}$ but since we are only integrating for the $0^{\circ}$ field we need to work on an oversized pupil to anticipate the effective clear aperture required by the full field of view of the system. Therefore we integrate $r$ from 0 to $40 \mathrm{~mm}$. To obtain tridimensional surfaces we introduce pupil polar coordinates $r$ and $\alpha$ given Eq. (9) and we perform the integration repetitively, for values of $\theta$ equally spaced between 0 and $2 \pi$ according Algorithm 2.

$$
p_{x}=r \cos \alpha \quad \text { and } \quad p_{y}=r \sin \alpha
$$

Function SolveParabasal:

Solve variables in the parabasal case (Table 1, col. 1). The solver used is SciPy [14]

implementation of Levenberg-Marquardt [15]. Variables marked derived are solved separately in closed-form.

Input: Variables marked set

Output: Variables marked solved or derived

Function Integrate:

The integration uses SciPy [14] implementation of a Runge-Kutta method [16] and calls a solve function similar to SolveParabasal with variables according Table 1, col. 2

Input: $\alpha$ and variables marked integrated or solved at $r$

Output: Variables marked integrated or solved at $r+\Delta R$

Algorithm 1: Functions definition 


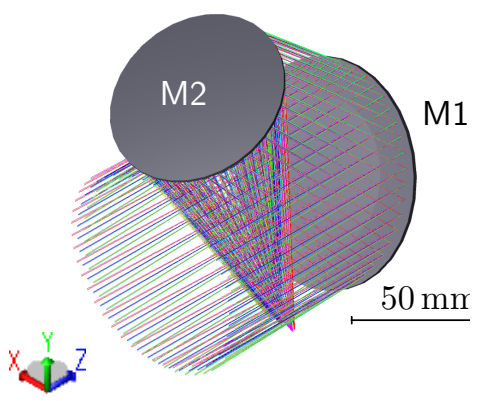

(a) Zemax view of the system, refocusing of $-517 \mu \mathrm{m}$ and FoV tilt of $\Delta \theta_{y}=0.168^{\circ}$

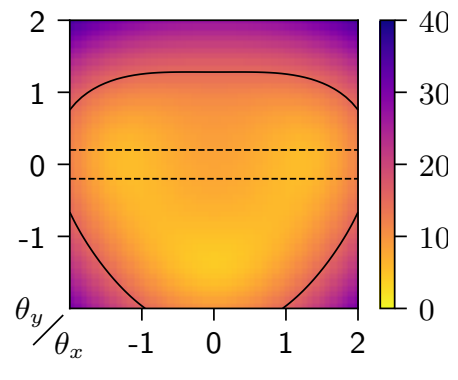

(b) Geometrical spot size, $50 \times 50$ fields, 120 rays per field in gaussian quadrature pattern, angles in degrees. Solid black: spot size $<15 \mu \mathrm{m}$, dashed: specified FoV

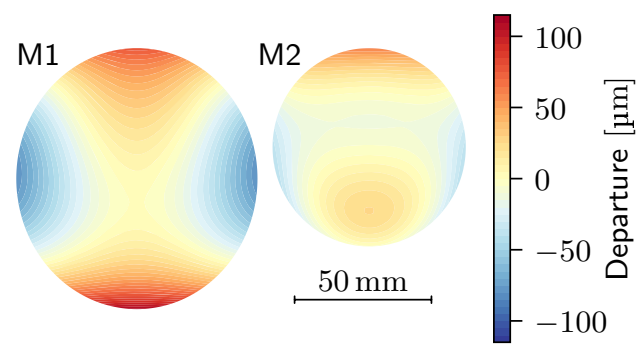

(c) Departure in the normal direction from a best fit sphere, M1 has a best fit radius of curvature of $799.21 \mathrm{~mm}$ and M2 of $377.18 \mathrm{~mm}$, both surfaces are concave.

Figure 3: Performance verification in Zemax and freeform surfaces maps.

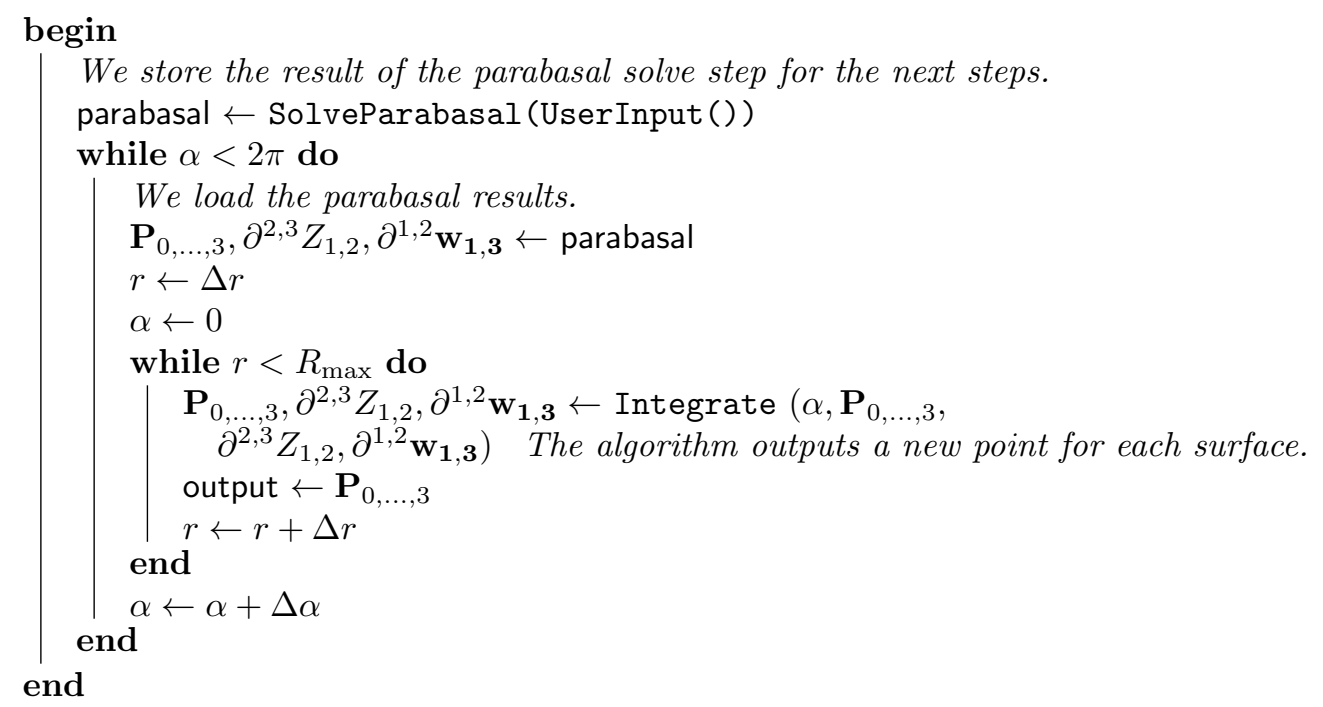

Algorithm 2: Integration algorithm, settings used: $\Delta \alpha=\pi / 100, \Delta r=1 \mathrm{~mm}, R_{\max }=40 \mathrm{~mm}$

After integration the profiles are interpolated on a grid $(200 \times 200$ points $)$ for importation in Zemax (Figure 3a). The optical system is refocalized and the FoV centered on the optimum direction by adjusting its orientation, this is necessary to overcome a shortfall of the algorithm which constructs a system with perfect axial stigmatism. By evaluating the RMS spot size performance in the field, we show that we obtain an asymmetric performance profile that allows to meet the requirement on a field with a triangular shape that encompass the rectangular specification (Figure $3 \mathrm{~b}$ ). The distortion evaluated on the same grid is below $0.48 \%$ for an achieved focal length of $149.7 \mathrm{~mm}$. We expect that a system with a larger field would exhibit significant distortion since no constraints were applied to this effect. The departures from a best fit sphere are shown in Figure 3c, both surfaces are concave which is an unusual combination for a telescope. No high-frequency ripples are observed, additionally the peak-to-valley departures are reasonable $(<200 \mu \mathrm{m})$ so we expect that these surfaces can be manufactured using single point diamond turning methods for infrared applications.

In this letter we have demonstrated a new method capable of constructing the freeform surfaces of a high- 
performance two-mirror freeform telescope without axial symmetry from only 5 degrees of freedom indicated in Figure 1, paving the way for a systematic exploration of the solution space. Future work on the method will concentrate on expanding to higher orders with the following expected improvements: suitability to systems with more than two freeform surfaces and the possibility to balance low and high order aberration terms, sacrificing if necessary axial stigmatism for an improved field performance. Additionally the method will be studied as a complement to classical optimization methods, generating starting points for problems with tight packaging constraints.

\section{References}

[1] Aaron Bauer, Eric M Schiesser, and Jannick P Rolland. Starting geometry creation and design method for freeform optics. Nature communications, 9(1):1756, 2018.

[2] Jean-Baptiste Volatier, Álvaro Menduiña-Fernández, and Markus Erhard. Generalization of differential ray tracing by automatic differentiation of computational graphs. JOSA A, 34(7):1146-1151, 2017.

[3] Harald Ries and Julius A Muschaweck. Tailoring freeform lenses for illumination. In Novel Optical Systems Design and Optimization IV, volume 4442, pages 43-51. International Society for Optics and Photonics, 2001.

[4] Juan C Miñano, Pablo Benítez, Wang Lin, José Infante, Fernando Muñoz, and Asunción Santamaría. An application of the sms method for imaging designs. Optics express, 17(26):24036-24044, 2009.

[5] Fabian Duerr, Pablo Benítez, Juan C Miñano, Youri Meuret, and Hugo Thienpont. Analytic free-form lens design in 3d: coupling three ray sets using two lens surfaces. Optics express, 20(10):10839-10846, 2012.

[6] Zhe Hou, Milena Nikolic, Pablo Benitez, and Florian Bociort. Sms2d designs as starting points for lens optimization. Optics Express, 26(25):32463-32474, 2018.

[7] Tong Yang, Guo-Fan Jin, and Jun Zhu. Automated design of freeform imaging systems. Light: Science Es Applications, 6(10):e17081, 2017.

[8] Tongtong Gong, Guofan Jin, and Jun Zhu. Point-by-point design method for mixed-surface-type off-axis reflective imaging systems with spherical, aspheric, and freeform surfaces. Optics express, 25(9):1066310676, 2017.

[9] GD Wassermann and E Wolf. On the theory of aplanatic aspheric systems. Proceedings of the Physical Society. Section B, 62(1):2, 1949.

[10] Donald Lynden-Bell. Exact optics: a unification of optical telescope design. Monthly Notices of the Royal Astronomical Society, 334(4):787-796, 2002.

[11] RV Willstrop and D Lynden-Bell. Exact optics-ii. exploration of designs on-and off-axis. Monthly Notices of the Royal Astronomical Society, 342(1):33-49, 2003.

[12] Bryan D Stone and Joseph M Howard. Low-order aberration coefficients of systems with freeform surfaces. Optical Engineering, 57(10):101702, 2018.

[13] Aaron Meurer, Christopher P. Smith, Mateusz Paprocki, Ondřej Čertík, Sergey B. Kirpichev, Matthew Rocklin, AMiT Kumar, Sergiu Ivanov, Jason K. Moore, Sartaj Singh, Thilina Rathnayake, Sean Vig, Brian E. Granger, Richard P. Muller, Francesco Bonazzi, Harsh Gupta, Shivam Vats, Fredrik Johansson, Fabian Pedregosa, Matthew J. Curry, Andy R. Terrel, Štěpán Roučka, Ashutosh Saboo, Isuru Fernando, Sumith Kulal, Robert Cimrman, and Anthony Scopatz. Sympy: symbolic computing in python. PeerJ Computer Science, 3:e103, January 2017. 
[14] Eric Jones, Travis Oliphant, Pearu Peterson, et al. SciPy: Open source scientific tools for Python, 2001-. [Online; accessed ;today i].

[15] Jorge J Moré. The levenberg-marquardt algorithm: implementation and theory. In Numerical analysis, pages 105-116. Springer, 1978.

[16] Ernst Hairer, Syvert P Nørsett, and Gerhard Wanner. Solving ordinary differential equations. i, volume 8 of springer series in computational mathematics, 1993. 